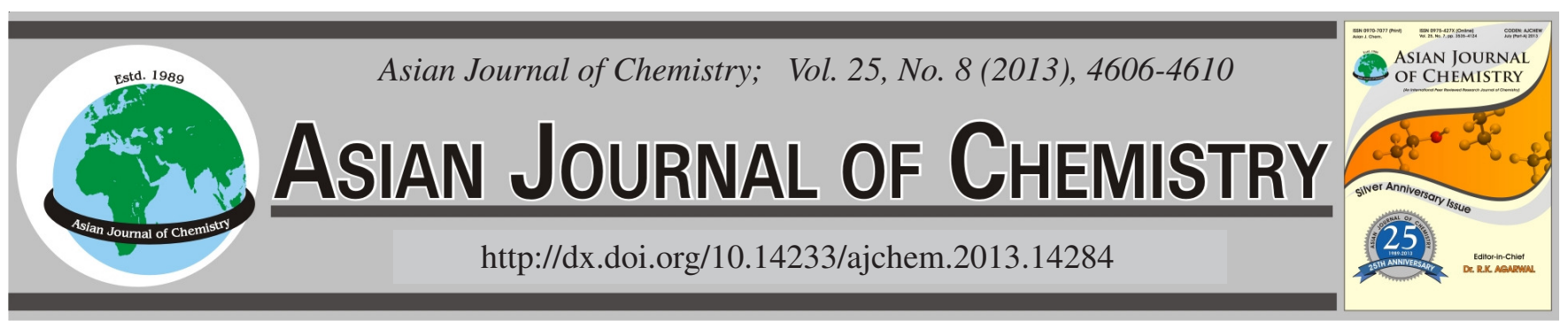

\title{
Adsorption Behaviour of Paeoniflorin on Two Kinds of Resins
}

\author{
Wei Zhang*, Qian Li ${ }^{1}$ and ShaOYAN WANG ${ }^{1}$
}

University of Science and Technology, Liaoning, Anshan 114044, Liaoning Province, P.R. China

*Corresponding author: Fax: +86 412 5929480; Tel: +86 412 5929030; E-mail: askdzw@163.com

(Received: 9 July 2012;

Accepted: 18 February 2013)

AJC-13023

\begin{abstract}
The adsorption characteristic of paeoniflorin was investigated in adsorption thermodynamics and kinetics of AB-8 and D101 resins in the static adsorption work. The experimental results showed that the adsorption of paeoniflorin on two resins belonged to monolayer adsorption and in accordance with Langmuir isotherm equation. The process of adsorption was in line with the first order kinetic equation. The adsorption process was exothermic and the adsorption behaviour was in nonlinear adsorption stage. All the adsorption process was ended in $1 \mathrm{~h}$. The adsorption behaviour of paeoniflorin on D101 was better than AB-8 resin. The results could be used to guide the separation experiments of paeoniflorin on macro porous resin.
\end{abstract}

Key Words: Paeoniflorin, Adsorption, Resin, Thermodynamics, Kinetics.

\section{INTRODUCTION}

Radix Paeoniae is the dried root out of skin which belongs to the ranunculaceae paeonia Lactiflora Pall and it has the ability of antiinflammatory, antiviral, solutions spasm, pain stopion and liver protection and other pharmacological activities $^{1}$. Paeoniflorin is the main active ingredient of Radix Paeoniae and belongs to the monoterpene glycosides. It has effects on lowing hypoglycemic ${ }^{2}$, protecting cardiovascular ${ }^{3}$, regulating the immune system ${ }^{4,5}$, etc.

Reports on the separation of paeoniflorin, the most commonly used mean is the macro porous resin. Adsorption resin is a polymer submitted globules which has an internal networklike structure with a choicable of pore structure and surface chemical structure, by intermolecular force organic compounds could be adsorbed from solution and then the purpose of separation could be achieved ${ }^{6}$. Therefore, the investigation of paeoniflorin in the adsorption behaviour on resin is good to the in-depth study of adsorption phenomenon. Provide valuable references for the adsorption resin on the separation of nature produces. In this study the regular pattern of adsorption thermodynamics and kinetics of paeoniflorin on solutions on resin is investigated which do some relevant theoretical exploration for the separate application of paeoniflorin on resins.

\section{EXPERIMENTAL}

Paeoniflorin standard sample (National Standard Material Center), Paeoniflorin extract for adsorption (made by Fine Separation of Engineering and Technology Center, University of Science and Technology of Liaoning, Content was more then $98 \%$ ), AB-8, D101 macro porous resins (Resin Technology Co., Ltd. Anhui Samsung), Reagents were analytical grade made in China.

Analysis methods: Shimadzu LC-10AT HPLC, SPD-10A UV detector, Agilent Column (ODS , $5 \mu \mathrm{m}, 4.6 \mathrm{~mm} \times 150$ $\mathrm{mm}$ ), flow rate: $U_{\mathrm{D}}=1.0 \mathrm{~mL} / \mathrm{min}, \lambda=230 \mathrm{~nm}$, mobile phase: alcohol:water $(\mathrm{V} / \mathrm{V})=30: 70$, injection volume was $20 \mu \mathrm{L}$.

Paeoniflorin standard curve: A certain amount of standard paeoniflorin was measured precisely and placed into a $50 \mathrm{~mL}$ volumetric flask. Then the volume was maintained with methanol and made up the concentration was $0.54 \mathrm{mg} / \mathrm{mL}$ of standard mixture solution respectively. Measured precisely standard mixture solution 1.0, 3.0, 5.0, 7.0 and $10.0 \mathrm{~mL}$ and placed in $10 \mathrm{~mL}$ volumetric flask, respectively. Compounding a series of concentration of the solution. The injected solutions were analyzed by HPLC. The ordinate was the peak area of reference substance and the abscissa was the concentration. Then draw the standard curve. It was concluded that paeoniflorin had a good liner relationship in the concentration of $0.054-$ $0.54 \mathrm{mg} / \mathrm{mL}$ and the regression equation was developed as follows: $\mathrm{Y}=207.8808 \mathrm{X}+4.9801, \mathrm{r}=0.9994$.

Pretreatment of the two resins and determination of settlement density: Took a certain amount of AB-8 and D101 resins, placed them in a watch glass respectively. Then covered with plastic wrap and holed a number of membrane with an awl in the preservation. The two resins were placed in vacuum drying oven and dried under vacuum at $333 \mathrm{~K}\left(60{ }^{\circ} \mathrm{C}\right)$. It indicated that the two resins had been dryed already when the weight of the two did not reduce after a certain of time. 
Pretreatment of resin: A certain quality of the two dried resins $(c a .1 \mathrm{~g})$ were left in constant with $95 \%$ ethanol $(10 \mathrm{~mL})$ for $2 \mathrm{~h}$ and soaked in deionized water for $20 \mathrm{~min}$, three times.

Determination of settlement density: The two pretreatmented resins were soaked with deionized water in graduated cylinder $(10 \mathrm{~mL})$. Measured the volume $(\mathrm{V})$ after resins were fully settlemented according to the formula $\rho=w / v$, the resin settlement density were calculated. The results were shown in Table-1.

TABLE-1

SETTLEMENT DENSITY OF TWO RESINS TABLE

\begin{tabular}{cccc}
\hline Resin type & $\begin{array}{c}\text { Quality of dry } \\
\text { resin }(\mathrm{g})\end{array}$ & $\begin{array}{c}\text { Volume of wet } \\
\text { resin }(\mathrm{mL})\end{array}$ & $\begin{array}{c}\text { Settlement } \\
\text { density }\left(\mathrm{kg} \mathrm{L}^{-1}\right)\end{array}$ \\
\hline $\mathrm{AB}-8$ & 1.0135 & 6 & 0.1689 \\
$\mathrm{D} 101$ & 0.9878 & 5 & 0.1976 \\
\hline
\end{tabular}

Effects of temperature on the adsorption capacity and adsorption rate of paeoniflution: Weighting accurately and pretreatmenting a certain amount of dried resins and left the resins in constant with a certain volume of adsorption solution and stirred in water bath for $20 \mathrm{~min}$ at the temperature of 293 , 298 and 303 K. Sealed and left the amount of paeoniflorin for $5 \mathrm{~h}$. The result of analysis suggests that the amount of paeoniflorin in supernatant until the supernatant concentration of paeoniflorin showed no change. (The rate of change $<3 \%$ ). Calculated value of the concentration of paeoniflorin according to the standard curve of paeoniflorin. The adsorption rate and adsorption capacity of paeoniflorin at different temperatures were calculated according to the formula (1), formula (2). The relationship between adsorption capacity and adsorption rate of resin could be obtained by:

$$
\begin{gathered}
q_{e}=\frac{\left(c_{0}-c_{e}\right) V_{0}}{M} \\
E(\%)=\frac{\left(c_{0}-c_{e}\right)}{c_{0}} \times 100 \%
\end{gathered}
$$

$\mathrm{q}_{\mathrm{e}}(\mathrm{mg} / \mathrm{g})$ is the equilibrium adsorption amount; $\mathrm{c}_{0}(\mathrm{~g} / \mathrm{L})$ is the paeoniflorin concentration in the initial solution; $c_{e}(g / L)$ is the adsorption equilibrium concentration of paeoniflorin; $\mathrm{V}_{0}$ (L) is the initial adsorption volume of paeoniflorin; $\mathrm{M}(\mathrm{g})$ is the quality of dry resin; $\mathrm{E}(\%)$ is the adsorption rate of resin adsorption.

The adsorption kinetics of paeoniflorin on two resins: Preparated five different concentration of paeoniflorin solution $50 \mathrm{~mL}$ and added two pretreatmented resins at the temperature of 293,298 and $303 \mathrm{~K}$. Then calculated the adsorption capacity correspondingly. The relationship between adsorption kinetics and themodynamics of paeoniflorin on two resins at different temperatures were investigated.

\section{RESULTS AND DISCUSSION}

Analysis about settlement density of two resins: In order to investigate the adsorption handling problem of two resins, we determined the settlement density of the two resins and results were shown in Table-1.

According to the Table-1, the settlement density of D101 was more than AB- 8 resin, so D101 was easer to fill in the resin column when filled the macro porous resin separation column and the settled time was shorter than $A B-8$ resin which saving the procession and separation time.

Effects of different temperatures on the adsorption of paeoniflorin on two resins: In order to investigate the adsorption behaviour on two resins of paeoniflorin, the experiment was studied by determining the equilibrium adsorption capacity and adsorption rate on two resins at three different temperatures. Draw three adsorption isotherm cures at different temperatures on two resins of paeoniflorin. The results were shown in Figs. 1-3 and Table-2. At the same time the data of Figs.1-3 and the Langmuir isotherm ${ }^{7}$ and Freundlich isotherm ${ }^{8}$ were fitted in Table-3.
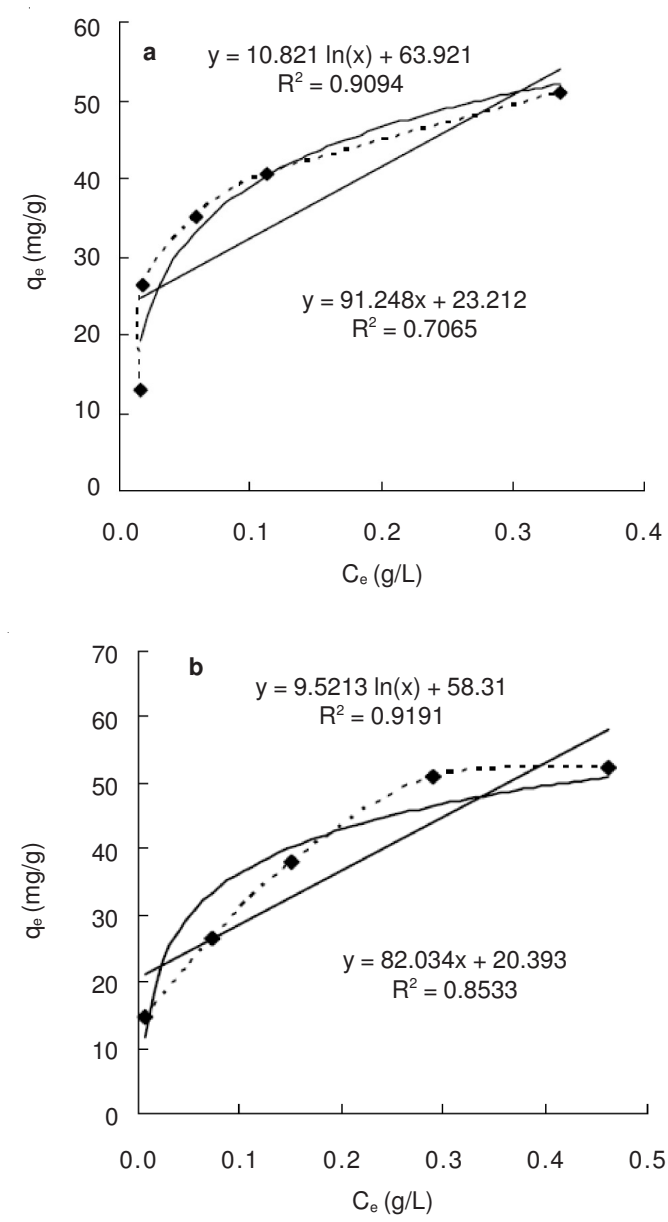

Fig. 1. Equilibrium adsorption isotherm for paeoniflorin on two resins at $293 \mathrm{~K}$ and fitted curve (a: AB-8, b: D101)

Table-2 and Figs. 1-3 showed that the adsorption capacity was enhanced differently by increasing the temperature, the adsorption process was endothermic process, while the adsorption capacity of D101 was significantly higher than AB-8 resin. It means that during choosing adsorption resins for paeoniflorin D101 was better than AB-8 resin.

Comparing the fitted results of Langmuir and Freundlich models, the correlation of regreision equation for Freundlich models was smaller than Langmuir models. It indicated that the adsorption of paeoniflorin on two resins followed Langmuir adsorption isotherm. Adsorption isotherms figures of two resins showed that the adsorption behaviour of paeoniflorin on both resins belonged to logarithmic adsorption not linar 
TABLE-2

ADSORPTION EQUILIBRIUM AND ADSORPTION RATE ON TWO RESINS AT THREE DIFFERENT TEMPERATURES

\begin{tabular}{lccc|cc}
\multicolumn{1}{c|}{ Resin type } & AB-8 & & D101 \\
\hline Temperature $(\mathrm{K})$ & 293 & 298 & 303 & 293 & 298 \\
Equilibrium adsorption capacity $\left(\mathrm{mg} \mathrm{g}^{-1}\right)$ & 20.56 & 21.13 & 23.90 & 26.51 & 27.61 \\
Resin adsorption rate (\%) & 98.79 & 97.28 & 97.19 & 98.74 & 99.00 \\
\hline
\end{tabular}

TABLE-3

FITTED EQUATION OF PAEONIFLORIN ON TWO RESINS FOR LANGMUIR ISOTHERM AND FREUNDLICH ISOTHERM

\begin{tabular}{cccccccc} 
Resin & $\mathrm{T}(\mathrm{K})$ & Langmuir & $\mathrm{R}^{2}$ & $\mathrm{q}_{\mathrm{m}}(\mathrm{mg} / \mathrm{g})$ & $\mathrm{K}_{\mathrm{L}}(\mathrm{L} / \mathrm{mg})$ & $\mathrm{R}^{2}$ \\
\hline \multirow{4}{*}{$\mathrm{AB}-8$} & 293 & $\mathrm{C}_{\mathrm{e}} / \mathrm{q}_{\mathrm{e}}=0.0177 \mathrm{C}_{\mathrm{e}}+0.0007$ & 0.9916 & 56.50 & 25.29 & $\log \mathrm{q}_{\mathrm{e}}=0.1225 \log \mathrm{C}_{\mathrm{e}}+0.829$ & 0.7671 \\
& 298 & $\mathrm{C}_{\mathrm{e}} / \mathrm{q}_{\mathrm{e}}=0.0152 \mathrm{C}_{\mathrm{e}}+0.0004$ & 0.9818 & 65.79 & 38 & $\log \mathrm{q}_{\mathrm{e}}=0.1359 \log \mathrm{C}_{\mathrm{e}}+0.8647$ & 0.9846 \\
& 303 & $\mathrm{C}_{\mathrm{e}} / \mathrm{q}_{\mathrm{e}}=0.0149 \mathrm{C}_{\mathrm{e}}=0.0003$ & 0.9893 & 67.11 & 49.67 & $\log \mathrm{q}_{\mathrm{e}}=0.1372 \log \mathrm{C}_{\mathrm{e}}+0.9116$ & 0.9441 \\
& 293 & $\mathrm{C}_{\mathrm{e}} / \mathrm{q}_{\mathrm{e}}=0.0171 \mathrm{C}_{\mathrm{e}}+0.001$ & 0.9781 & 58.48 & 17.10 & $\log \mathrm{q}_{\mathrm{e}}=0.108 \log \mathrm{C}_{\mathrm{e}}+0.8692$ & 0.9779 \\
\hline \multirow{2}{*}{$\mathrm{D} 101$} & 298 & $\mathrm{C}_{\mathrm{e}} / \mathrm{q}_{\mathrm{e}}=0.0172 \mathrm{C}_{\mathrm{e}}+0.0008$ & 0.9835 & 58.14 & 21.50 & $\log \mathrm{q}_{\mathrm{e}}=0.1066 \log \mathrm{C}_{\mathrm{e}}+0.8958$ & 0.9766 \\
& 303 & $\mathrm{C}_{\mathrm{e}} / \mathrm{q}_{\mathrm{e}}=0.0145 \mathrm{C}_{\mathrm{e}}+0.0004$ & 0.9876 & 68.97 & 36.35 & $\log \mathrm{q}_{\mathrm{e}}=0.1279 \log \mathrm{C}_{\mathrm{e}}+0.9116$ & 0.9695 \\
\hline
\end{tabular}
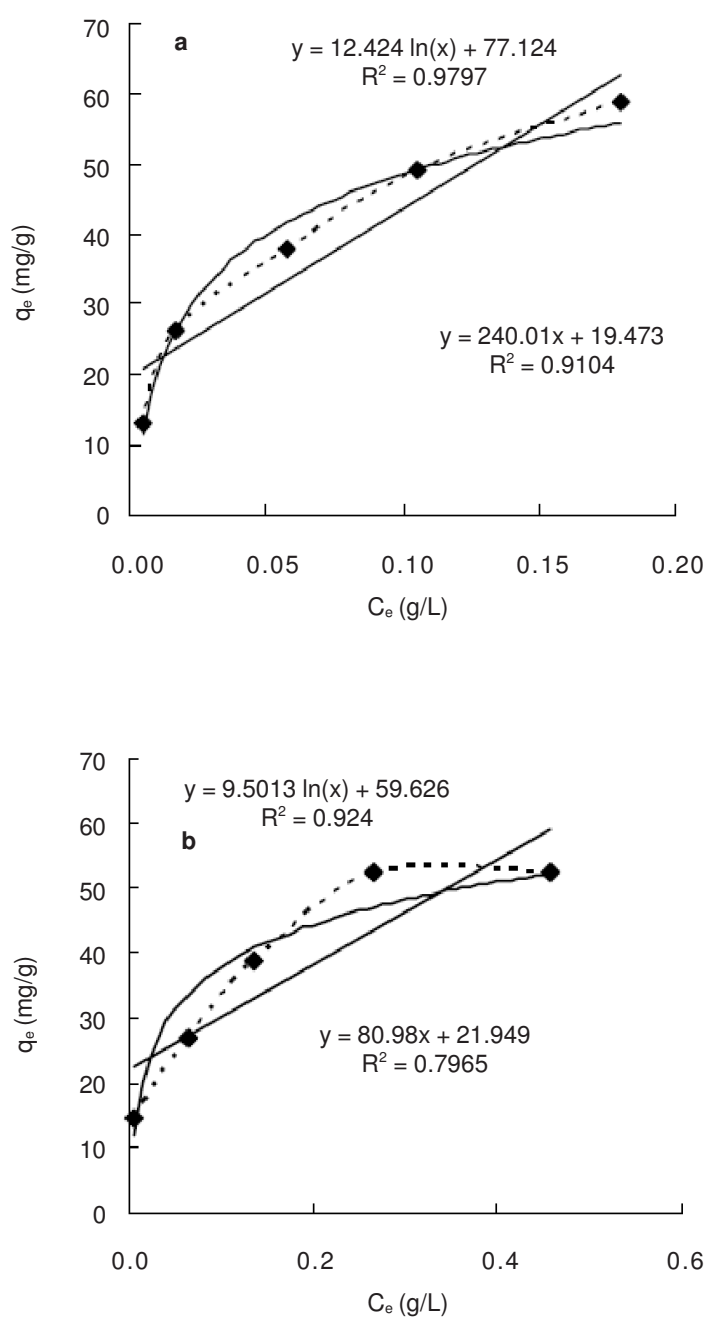

Fig. 2. Equilibrium adsorption isotherm for paeoniflorin on two resins at $298 \mathrm{~K}$ and fitted curve (a: AB-8, b: D101)

adsorption in the experimental concentration range. It also showed that in the experimental range of concentration the paeoniflorin concentration was in the non-linear range of the adsorption solution. It had a reference value of paeoniflorin when used the resin column at the non-linear conditions and from the two linear fitted curve of resins. It is concluded that the coefficient $\mathrm{K}$ by examing fitted straight lines of each resin
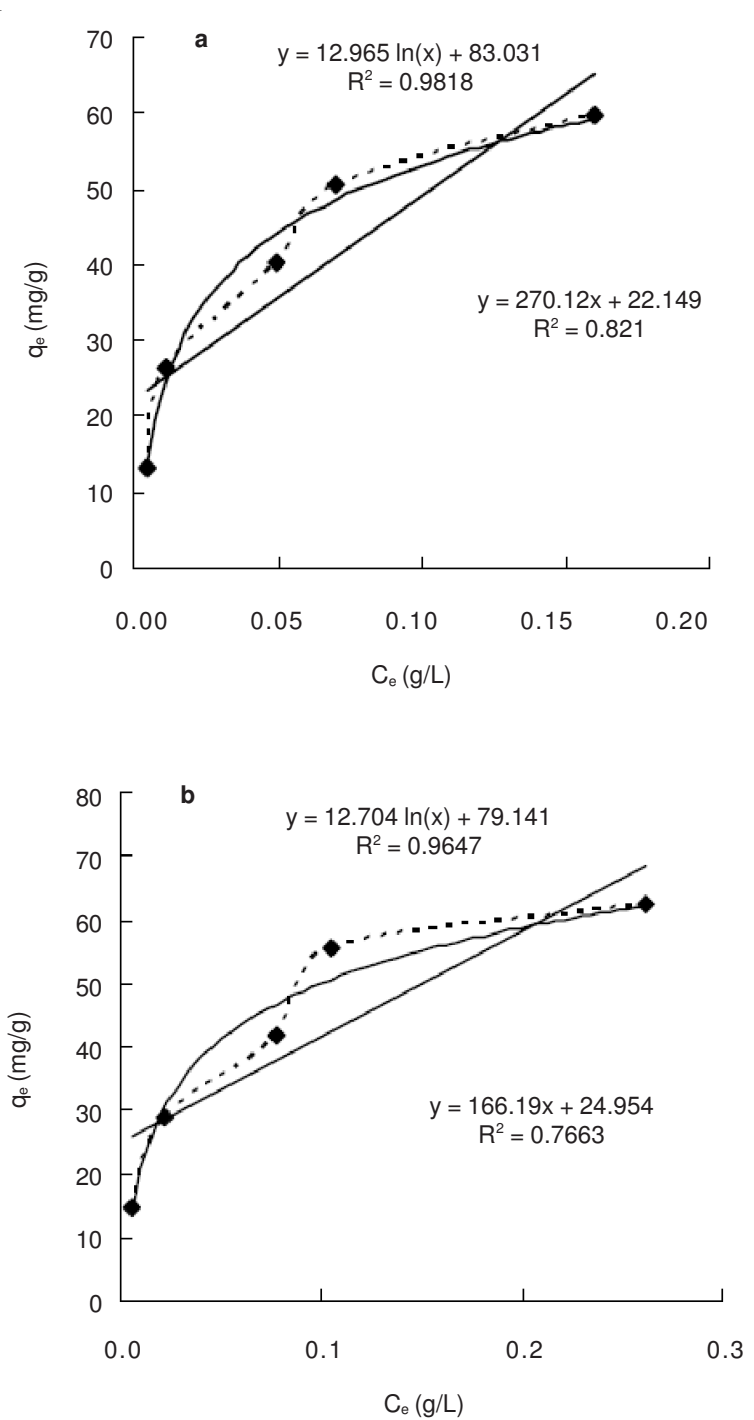

Fig. 3. Equilibrium adsorption isotherm for paeoniflorin on two resins at $303 \mathrm{~K}$ and fitted curve (a: AB-8, b: D101)

adsorption isotherm at different temperatures. By examing the coefficient $\mathrm{K}$ of two resins at different temperatures from Table3 , we know coefficient $\mathrm{K}$ enhanced by increasing the temperature which illustrates that the two resins for the adsorption behaviour of paeoniflorin were endothermic behaviour. At the 
same time compared the largest signal- adsorption calculations of the two resins and it can be noticed that the maximum adsorption capacity of D101 was higher than AB-8 which was the same with experimental results and it also showed that D101 was indeed better than the AB-8 resin in the aspect of adsorpting paeoniflorin.

Adsorption kinetics of paeoniflorin on two resins: The adsorption time can be used as the abscissa and the adsorption capacity of paeoniflorin in the adsorption solution on two resins at different times can be used as the longitudinal coordinates. Then the static adsorption curves of the two resins at three kinds of temperatures can be drawn (Fig. 4).
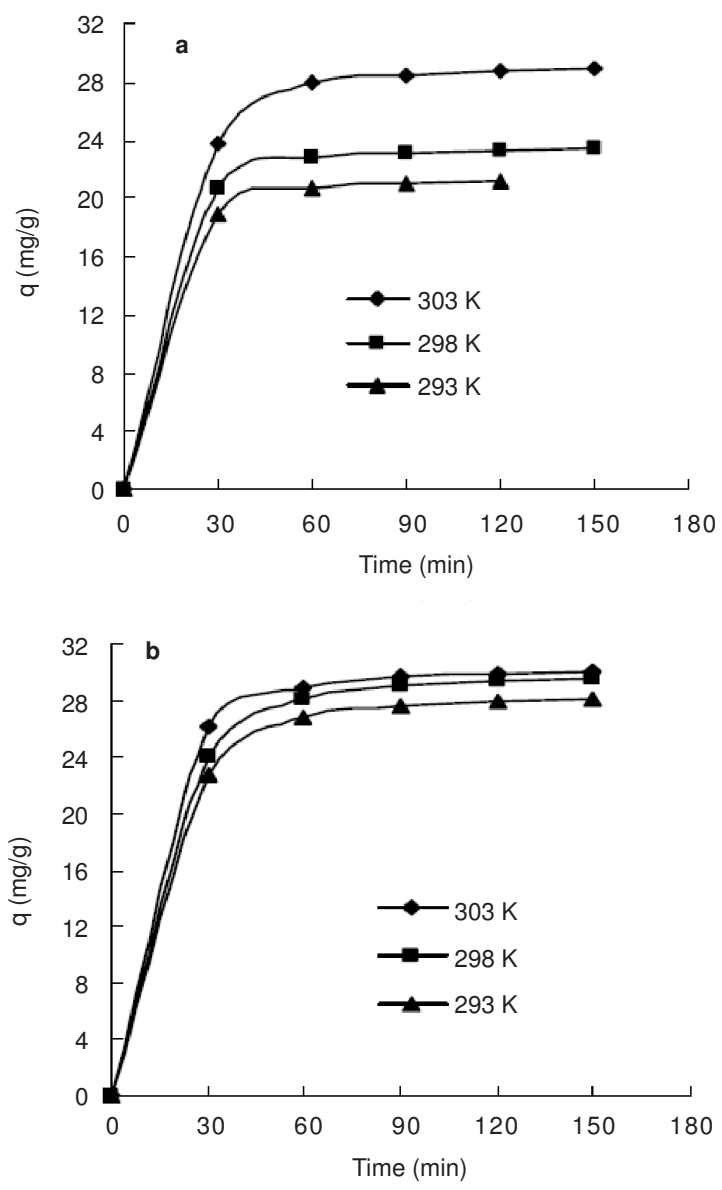

Fig. 4. Static adsorption curve of paeoniflorin on two resins at different temperatures (a: AB-8, b: D101)

The adsorption capacity (q) changes with the passage of time according to a curve. From the curve it is concluded that in the initial stage the curve increased steeply and the adsorption rate was very high. 0-30 min was the rapidly adsorption stage, $0.5-1.0 \mathrm{~h}$ was the stage of no adsorption saturated, $1 \mathrm{~h}$ and longer was the stage of full adsorption. So in the separation of paeoniflorin on two resins experiment we can conclude that the sampling had an effect on determining the separation conditions.

Described the adsorption dynamic behaviour of paeoniflorin on two resins by standard first order kinetics model (Lagergren isotherm) ${ }^{9}$ and the quasi-second order linetics model ${ }^{10}$. The results were shown in Figs. 5 and 6, and Table-4.
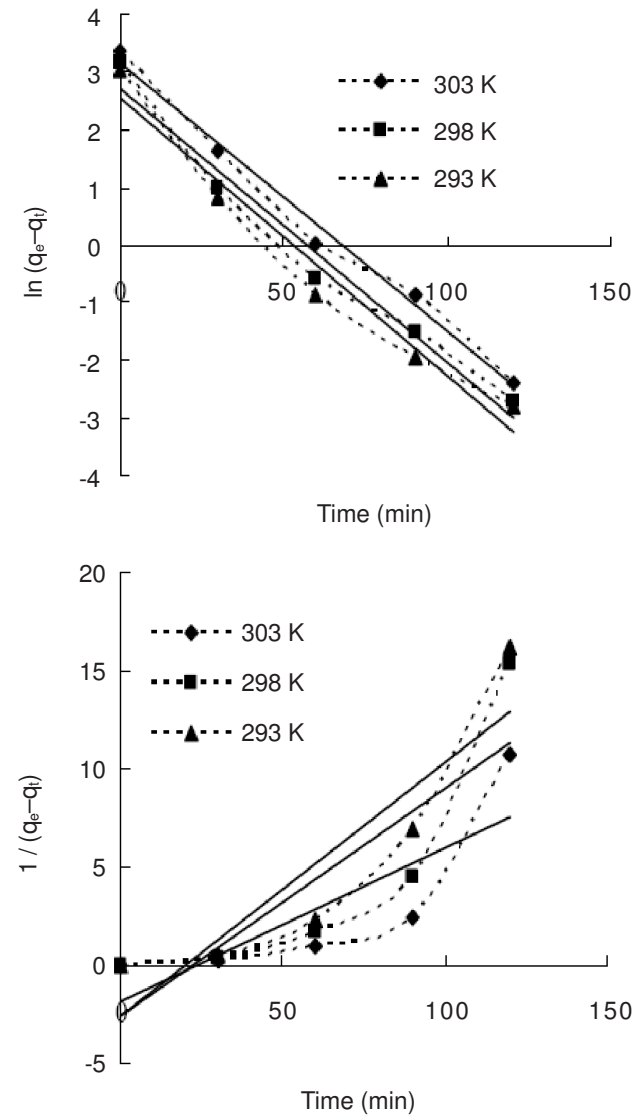

Fig. 5. Adsorption dynamic curve of resin AB-8 towards paeoniflorin at different temperatures
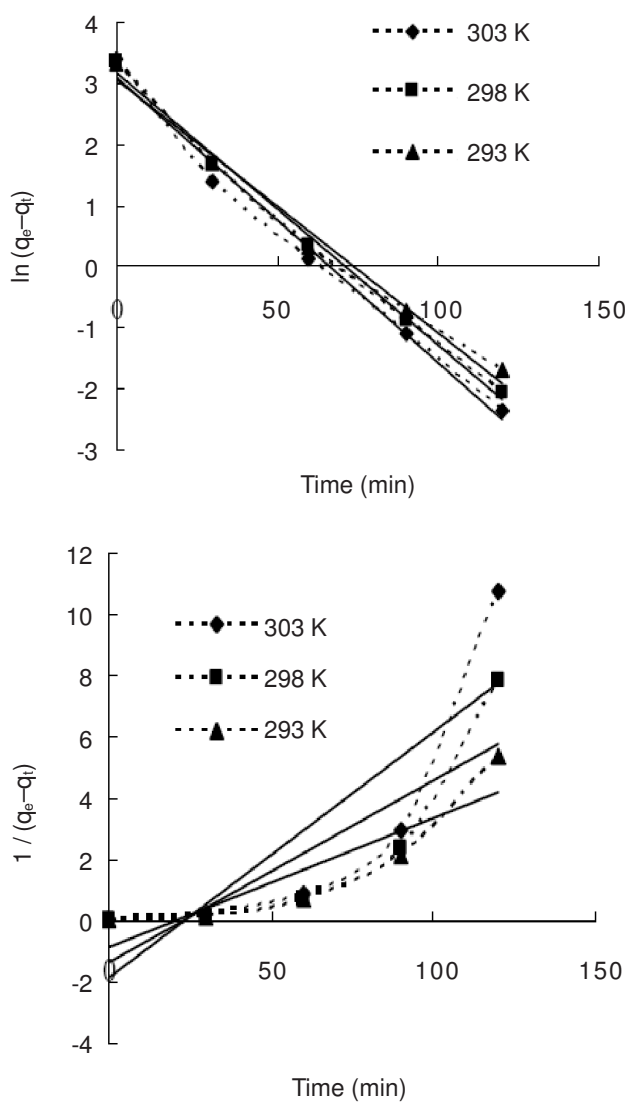

Fig. 6. Adsorption dynamic curve of resin D101 towards paeoniflorin at different temperatures 


\begin{tabular}{cccccc}
\hline \multicolumn{6}{c}{ TABLE-4 } \\
& $\begin{array}{c}\text { ADSORPTION KINETICS OF PAEONIFLORIN ON } \\
\text { TWO RESINS AT DIFFERENT TEMPERATURES }\end{array}$ \\
\hline \multirow{2}{*}{ Resin } & \multirow{2}{*}{$\ln \left(\mathrm{q}_{\mathrm{e}}-\mathrm{q}_{\mathrm{t}}\right)=\ln \mathrm{q}_{\mathrm{e}}-\mathrm{K}_{\mathrm{l}} \mathrm{t}$} & \multicolumn{1}{l}{$1 /\left(\mathrm{q}_{\mathrm{e}}-\mathrm{q}_{\mathrm{t}}\right)=1 / \mathrm{q}_{\mathrm{e}}+\mathrm{K}_{2} \mathrm{t}$} \\
\cline { 3 - 6 } & & $\mathrm{K}_{1}(1 / \mathrm{min})$ & $\mathrm{R}^{2}$ & $\mathrm{~K}_{2}$ & $\mathrm{R}^{2}$ \\
\hline \multirow{3}{*}{$\mathrm{AB}-8$} & 293 & 0.048 & 0.9621 & 0.0786 & 0.6892 \\
& 298 & 0.0476 & 0.9746 & 0.1161 & 0.7472 \\
& 303 & 0.04661 & 0.9893 & 0.1296 & 0.8313 \\
\hline \multirow{3}{*}{$\mathrm{D} 101$} & 293 & 0.0467 & 0.9863 & 0.042 & 0.8059 \\
& 298 & 0.0449 & 0.9946 & 0.0594 & 0.7401 \\
& 303 & 0.0416 & 0.9882 & 0.0804 & 0.7195 \\
\hline
\end{tabular}

It can be seen from Table-4 that the adsorption kinetics of paeoniflorin on two resins meet with the standard first-order kinetics $\left(R^{2}>0.96\right)$ and at the same time the constant of adsorption $\mathrm{K}_{1}$ was decreased by increasing the temperature. It is concluded that the increasing temperature did good to the strengthen of diffusion, it was not easy to reach the adsorption equilibrium of resins and the adsorption rate decreased. According to the adsorption equilibrium figures above, we also knew that it needed a little long time to reach the adsorption equilibrium with the increasing of temperature but the change not obvious.

Adsorption thermodynamics of paeoniflorin on two resins: From the adsorption isotherm equation it is clear that the adsorption behaviour on two resins of paeoniflorin were consistent with Langmuir isotherm equation. The coefficient of $\mathrm{K}_{\mathrm{L}}$ in Langmuir isotherm equation changed with the changing of temperature and adsorption heat. And their relationship can be illustrated as follows: $\ln \left(\mathrm{K}_{\mathrm{L}}\right)=\ln \left(\mathrm{K}_{0}\right)+(-\Delta \mathrm{H} / \mathrm{RT})^{11}$. Adsorption enthalpy can be derived from the equation, where $\Delta \mathrm{H}$ is the adsorption enthalpy for the same amount. $\mathrm{R}$ is the ideal gas constant $(8.314 \mathrm{~J} / \mathrm{mol} \mathrm{K}) . \mathrm{K}$ is the absolute temperature. $\Delta \mathrm{H}$ by $\ln \left(\mathrm{K}_{\mathrm{L}}\right)$ of $1 / \mathrm{T}$ plot of the slope obtained, According to the Gibbs equation the adsorption free energy change is $\Delta \mathrm{G}=$ -RT $\ln \mathrm{K}^{12}$. In the formula $\mathrm{K}$ is equal to the equilibrium adsorption coefficient $\mathrm{K}_{\mathrm{L}}$. The energy change associates with adsorption can be calculated by Gibbs-Helmholtz equation $(\Delta \mathrm{G}=(\Delta \mathrm{H}-\Delta \mathrm{G}) / \mathrm{T}$. The calculated equivalent adsorption enthalpy free energy and entropy changes were shown in Table-5.

TABLE-5

ADSORPTION THERMODYNAMICS DATA OF PAEONIFLORIN ON TWO RESINS AT DIFFERENT TEMPERATURES

\begin{tabular}{cccccccc}
\hline \multirow{2}{*}{ Resin } & $\Delta \mathrm{H}$ & \multicolumn{4}{c}{$\Delta \mathrm{G}(\mathrm{KJ} / \mathrm{mol})$} & & \multicolumn{2}{c}{$\Delta \mathrm{S}(\mathrm{KJ} / \mathrm{mol} \mathrm{K})$} \\
\cline { 3 - 8 } & $(\mathrm{KJ} / \mathrm{mol})$ & $293 \mathrm{~K}$ & $298 \mathrm{~K}$ & $303 \mathrm{~K}$ & $293 \mathrm{~K}$ & $298 \mathrm{~K}$ & $303 \mathrm{~K}$ \\
\hline AB-8 & 49.87 & -7.87 & -9.01 & -9.84 & 0.197 & 0.198 & 0.197 \\
$\mathrm{D} 101$ & 55.33 & -6.92 & -7.60 & -9.04 & 0.212 & 0.211 & 0.213 \\
\hline
\end{tabular}

Table- 5 showed the enthalpy change was positive which indicated that the adsorption process was endothermic. Free energy changed greatly at different temperatures and the reasonable explanations can be given as follows: the adsorption behaviour was in the nonlinear adsorption stage and the concentration of paeoniflorin was very high in the paeoniflorin adsorption and separation. The free energy was negative which reflected the behaviour of adsorption media tends to the adsorption from the solution. The entropy was positive which indicated the adsorption process of paeoniflorin to two resins was in consistent with the greater degree of freedom in the near-saturating of the solution.

\section{Conclusion}

The behavioural characteristics of the adsorption kinetics and adsorption thermodynamics of paeoniflorin on two resins had been studied by examining the static adsorption experiments of paeoniflorin on AB-8 and D101 resins. The equilibrium adsorption capacity of paeoniflorin on D101 resin is larger than $\mathrm{AB}-8$ resin and the equilibrium adsorption capacity raised with the temperature increasing, but little increased in the degree. The adsorptions of paeoniflorin on two resins were consistent with Langmuir adsorption equation and id belonged to the non-linear adsorption. Thermodynamic behaviour indicated that the adsorption process was endothermic process and the free energy changed greatly at different temperatures which mainly due to the adsorption behaviour was in the nonliner adsorption stage. The results can provide scientific reference for the separation of paeoniflorin on resins column.

\section{REFERENCES}

1. X.Y. Zhang, C.K. Gao, J.H. Wang et al., Acta Pharm. Sin., 37, 305 (2002).

2. M. Kaneda, Y. Iitaka and S. Shibata, Tetrahedron, 28, 4309 (1972).

3. R. Sun, L.L. Lv, S.D. Guo and G.Q. Liu, J. Harbin Univ. Commerce, 21, 408 (2005).

4. A. Sugaya, T. Suzuki, E. Sugaya, N. Yuyama, K. Yasuda and T. Tsuda, J. Ethnopharmacol., 33, 159 (1991).

5. T. Okubo, F. Nagai, T. Seto, K. Satoh, K. Ushiyama and I. Kano, Biol. Pharm. Bull., 23, 199 (2000).

6. F. Gao, W. Yan and M. Shi, Appl. Chem. Ind., 39, 806 (2010).

7. M.H. Fan and S.Y. Xu, Sep. Purif. Technol., 61, 211 (2008).

8. Y.J. Fu, Y.G. Zu, W. Liu, C.L. Hou, L.Y. Chen, S.M. Li, X.G. Shi and M.H. Tong, J. Chromatogr. A, 1139, 206 (2008).

9. S. Lagergren, Handlingar, 24, 1 (1898)

10. W.J. Weber Jr. and J.C. Morris, Sanit Eng. Div. Am. Soc. Civ. Eng., 89, 31 (1963).

11. R.A. Garcla-Delgado, Sep. Sci. Technol., 27, 975 (1992).

12. P.B. John and T. Marios, J. Water Pollut. Control Fed., 59, 191 (1987). 